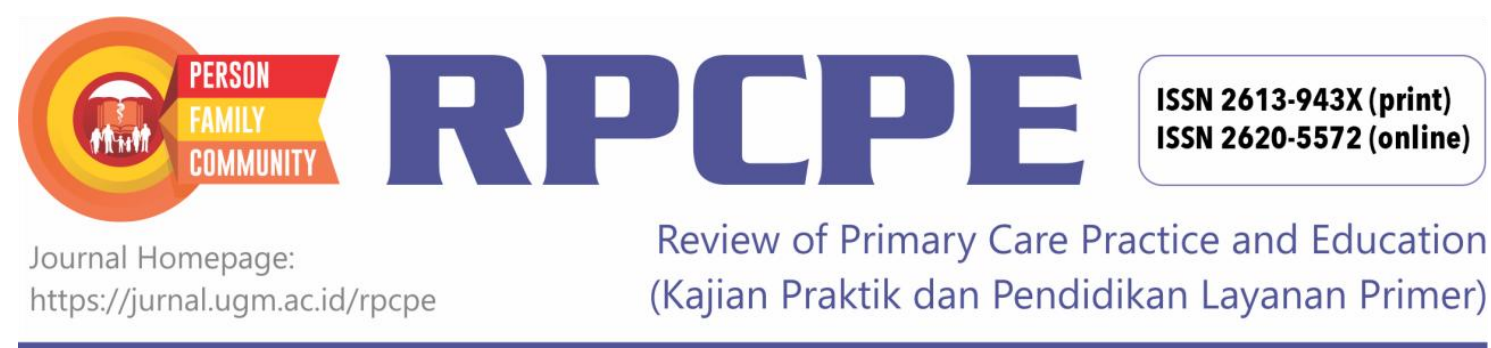

\title{
Description of Patient Acceptance of Use of Mask by Doctor at Poly Out-Patient Care Puskesmas, Bantul
}

\author{
Wahyu Pamungkasih ${ }^{1}$, Adi Heru Sutomo ${ }^{2}$, Mahar Agusno ${ }^{3}$ \\ ${ }^{1}$ Puskesmas Banguntapan II; Yogyakarta; Indonesia \\ ${ }^{2}$ Department of Family and Community Medicine; Faculty of Medicine, Public Health, and Nursing;Universitas Gadjah Mada; \\ Indonesia \\ ${ }^{3}$ Department of Psychiatry; Faculty of Medicine, Public Health and Nursing; Universitas Gadjah Mada; Indonesia \\ Corresponding Author: \\ Wahyu Pamungkasih: Puskesmas Banguntapan II, Jl. Pasopati No. 99, Krobokan, Tamanan, Banguntapan, Bantul, Daerah Istimewa \\ Yogyakarta - 55191, Indonesia \\ E-mail: pamungkasihwahyu@gmail.com
}

To cite this article:

Pamungkasih W, Sutomo AH, Agusno M. Description of patient acceptance of use of mask by doctorat poly out-patient carepuskesmas, Bantul. Rev Prim Care Prac and Educ. 2019; 2(2): 70-75.

\begin{abstract}
Background: The use of masks affects doctor and patient communication verbally and nonverbally. Objectives: This study aims to describe the patients' acceptance of the use of surgical masks by doctors in the patient's hearing, trust, and closeness. Methods: The research used qualitative design with phenomenological approach. In-depth interviews were conducted with 18 outpatients. Data were analyzed by content analysis. Results: Patient acceptance will be good when patients have the concept that masks are worn as a uniform and according to rules to prevent disease transmission. The use of masks does not affect the closeness and trust of patients even though they reduce hearing ability of patients, especially elderly patients. Still, they do not always ask the doctor about what is unclear. Some patients consider masks to be useful for preventing smelling of patients. Conclusions: The use of masks by doctors is acceptable to patients, does not affect the trust and closeness of patients even though it reduces hearing ability in certain patients.
\end{abstract}

Keywords: closeness, masks, non-verbal communication, patient-doctor communication, trust verbal communication

\section{BACKGROUND}

Consultation is at the core of the doctor's service activities ${ }^{1}$. In this consultation, there is communication between doctors and patients in an effort to establish therapeutic relationships. Communication with patients is an art in the practice of medicine ${ }^{2}$. Communication by the patient's doctor can be done verbally and non-verbally. Nonverbal communication is communication that is delivered not in the form of pure language context but through body gesture, touch, body position, facial expressions, eye contact, manner of dress and hairdo ${ }^{3}$. Fifty-five percent of doctor-patient communication is delivered through nonverbal communication ${ }^{4}$.

The patient's inability to understand what the doctor is saying will be a barrier to doctor-patient communication. The obstacle of doctor-patient communication can occur due to the doctor's communication skills and patient factors such as the patient's level of education ${ }^{5}$. Patient complaints about doctors are usually more often related to communication problems, not to the doctor's technical competency $6,7,8$.

Doctor's empathy is the socio-emotional competence in how the doctor shows understanding about the patient's situation, namely the perspectives, beliefs, experiences and understanding of the patient then communicates that understanding correctly with feedbacks to the patient, and reacts to the patient's understanding of the therapeutic concepts $^{3,9}$. Doctor's empathy is influenced by various factors of non-verbal verbal communication by doctors.

Barriers to nonverbal communication can occur due to physical barriers, for example, due to the use of masks by doctors which make the facial expressions of doctors not be fully visible to patients. One study found that the use of masks by doctors during consultations had a negative influence on the empathy of doctors and reduced ongoing 
relationships between physician and their patients ${ }^{10}$.

Masks are one of the personal protective equipment that is often used by doctors. Surgical masks are one of the personal protective equipment used in the concept of Universal Health Awareness. Health workers are at risk of transmitting disease from patients ${ }^{11,12}$. However, patient comfort will be reduced due to the use of masks by health workers, because patients cannot see the overall facial expression of the doctor ${ }^{13,14}$. The use of masks by doctors during consultation has a negative influence on the empathy of doctors and decreases the ongoing relationship between physician patients but is not related to overall patient satisfaction ${ }^{15}$

This study aimed to determine what is the description of patient acceptance of the use of masks by doctors in Poli Rawat Jalan related to the acceptance of hearing, closeness and trust felt by patients to doctors?

\section{RESEARCH METHODS}

This study was a qualitative research with phenomenological approach. The researchers collected data by in-depth interviews with outpatients served by doctors who used surgical masks continuously during the service process.

The inclusion criteria of this study were patients over 18 years of age and Javanese culture. Data were analyzed by content analysis and triangulated with three coders to maintain data validity. The coders are Master of Family Medicine students who have the same ability in patientdoctor communication. This research has obtained an Ethical Clearance from the Ethics Committee of the Faculty of Medicine UGM. There is no mask sponsor in this study.

\section{RESULTS AND DISCUSSION}

The study was conducted with 18 study participants who met the inclusion and exclusion criteria with the characteristics of the research subjects as follows:

Table 1. Characteristics of research participants

\begin{tabular}{lcc}
\hline & Characteristic & n \\
\hline Gender & Male & 8 \\
Age & Female & 10 \\
& $>55$ y.o & 6 \\
& $45-55$ y.o & 3 \\
Education & $31-44$ y.o & 6 \\
& $18-30$ y.o & 3 \\
Income & to JHS & 7 \\
The background is close to health workers & SHS & 6 \\
Guarantee ownership & $\geq$ D3 & 5 \\
& < Regional Minimum Salary & 7 \\
& Regional Minimum Salary & 11 \\
& Yes & 7 \\
& No & 11 \\
& JKN PBI & 6 \\
Reason to come & JKN Non-PBI & 7 \\
& JKN Mandiri & 2 \\
& Another guarantee & 2 \\
& None & 1 \\
& Routine control & 7 \\
& Sick & 10 \\
& Looking for healthy letters & 1 \\
\hline
\end{tabular}

There are 4 categories that describe the experience of patient admission when served by a doctor who wears a mask in an outpatient service. There is the category of verbal communication of doctors with sub-categories of hearing. The second and third categories are the feeling of the patient's closeness and the patient's trust in the doctor. The fourth category about the patient's concept of using masks has four sub-categories, namely masks, as a rule, useful in preventing disease transmission, the benefits of preventing dust and odor.

Participant 2 said the doctor's voice volume became less loud for patients who are elderly. The sound component becomes important in the reception of the sense of hearing. It was also similarly stated by participants 6 and 18 who felt their hearing was reduced due to age. Following the quotation:

"Because our hearing is lacking, sorry I can't see, he says less, it's unclear.” (Participant 6)

"I chose those who did not use masks because it was clear to speak. I was called but didn't hear." (Participant 18)

Verbal communication delivered by the doctor will be captured by the patient's sense of hearing. There are several factors that influence whether voice messages can be well-received, involving both the message giving factor and the recipient. Patient acceptance, when served by a doctor wearing a mask, is also influenced by the patient's background or characteristics such as gender, age, and guarantee ownership ${ }^{16}$. This should be noted for doctors who wear masks and serve elderly patients.

A patient's hearing reception becomes less clear when doctors use masks because doctors sometimes mumble and patients cannot see the doctor's lips and facial expressions 
as reflected in the following quotations:

"Yes, like mumbling." (Participant 10)

"Yeah, if you don't wear a mask, you can see and also clarify the conversation. The pronunciation is not clear. Yes, the conversation is not clear." (Participant 12)

As explained, the first step in diagnosis is to conduct medical interviews with patients. Nonverbal communication such as visual communication, body position, head movements, distance with patients, facial and voice expressions are related to patient satisfaction. The patients' complaints, $70 \%$, to doctors include less attention to patients, consider patient comments to be worthless, provide incomplete information to patients, lack empathy for patients and cannot understand them ${ }^{8}$.

The patient's inability to understand what the doctor is saying will be a barrier to doctor-patient communication ${ }^{5}$. Some patients who experience impaired hearing reception because of the use of masks by doctors say they don not always ask the doctor about what is unclear. As with participant 6 who is 66 years old with Javanese cultural characteristics, some patients chose not to ask and try to understand on their own like the following quote:

"Yes, it doesn't matter, but sometimes we understand at a glance, oh it means this, we already understand. We already understand. Just like that one ... just one or two words." (Participant 6)

Even though Yogyakarta has different generations, the Javanese people who live in DIY have the same ways and principles in expressing emotions. Like other Asian societies, Javanese cultures prioritize balance and harmony in human relations, and they tend not to express excessive emotional responses either through words or facial expressions. To avoid conflict they choose to avoid situations that cause emotional responses. The DIY community is also very careful in expressing and interpreting non-verbal communication ${ }^{17}$.

Another case involves participant 7 who chose to estimate what was not clear from the hearing she received due to fear to ask the doctor. Participant 7 is a female patient aged 19 years, with low economic status and had recently graduated from a formal school. Additionally, her limited experience of communicating with many people can be a factor in the patient's fear of the doctor.

In some Asian cultures, doctors still hold full authority in therapeutic decisions and override input from patients ${ }^{18}$. This is what makes some patients place themselves inferior to doctors so that it is difficult to express complaints and opinions during the discussion stage with their doctor.

Doctors need to confirm their understanding often when dealing with patients with characteristics that are inferior to doctors because of various factors such as education and a low economy. Barriers to patient-doctor communication can occur due to patient education ${ }^{18}$.
Other participants said that doctor-patient communication was affected by the patient's hearing even though it was not extensive because it was influenced by other factors such as other activities performed by doctors such as writing or because of the crowded environmental conditions due to many patients. One patient advised the doctor against using a mask to clarify the conversation especially when the condition of the health facility is crowded or when explaining the patient's illness or medication. The following is a quotation:

"Yes, maybe from the outside, yes, the problem is sometimes if the patient room is crowded, there are many people who talk that causes communication to be rather unclear. Sometimes the doctor writes while talking. Sometimes it's not clear, but still, do not understand. So, it's a little less vague." (Participant 16)

Related to this, spatial planning and the number of patients in health services must be considered so that the atmosphere of the health service environment becomes more comfortable and not noisy. But when considering Javanese culture that maintains harmony and maybe because of the patient's education level, not all patients will ask the doctor to clarify because they do not dare to ask the doctor and just guess about the meaning for themselves. The following is a related quote:

"I just guess. I hesitate to ask." (Participant 7)

"I just ask for a repeat explanation." (Participant 12)

The other participants said there was no effect on the use of masks with received hearing. As quoted by Ong et al., that doctor-patient communication is not all received through verbal language. Only 7\% were delivered verbally and 22\% with voice intonation. Meanwhile 55\% of communication is delivered through non-verbal communication both from eye contact, facial expressions, body position, voice intonation, etc ${ }^{19}$.

Research on communication between health officers states that the problem of communication in outpatient care is not so complicated, because not so many officers are involved. However, a high workload of doctors can affect the mood of officers, including doctors, which affects verbal and non-verbal communication ${ }^{20}$.

One study of interpersonal communication of patients at the Bethesda Hospital in Tomohon in outpatient / obstetric polyclinic installations revealed that barriers to language barriers can be obstacles to the verbal communication process of patient and doctors ${ }^{21}$. In this study, the culture of doctors and patients is the same, namely the Javanese culture so that it supports verbal communication and does not interfere with communication even though doctors wear masks.

Patient's perceived resistance to non-verbal communication due to the use of masks is an influence on closeness with the doctor. Research by Wong et al. found that the use of masks by doctors during consultations had a negative impact on the empathy of doctors and reduced the positive effects of 
relationships that had been established sustainably ${ }^{10}$.

If the patient has difficulty to express their complaints, the history done by the doctor will not be effective so that the information is incomplete. Information from the historical results will be one of the bases for establishing a patient diagnosis. If the information extracted is incomplete, the patient's diagnosis becomes less comprehensive and inaccurate.

Participants with a higher education level in this study revealed that they had known the concept of masks in preventing disease transmission. However, they have reduced patient and doctor closeness with the use of masks. Participants 3 who was educated at D3 hospital administration, 4, 11 and 15 who were scholars and 7 participants who were educated at Vocational School said the patient's doctor's relationship became less familiar with masks.

Even participant 15 was self-conscious whether the patient smelled bad when the doctor was wearing a mask, as quoted below:

"... the doctor is wearing a mask, it seems like the patient actually has a smelly disease or what, I mean it doesn't matter, the doctor's closeness with the patient is like stunted."

Some patients have the concept that masks are useful for preventing odors. As expressed by participants 2 and 8 who have low educational backgrounds. The quotations are as follows:

"If the mask is to prevent odor." (Participant 2)

"The first, mask is useful to prevent the dust and odor." (Participant 8)

The patients' concept of the use of masks needs to be improved because if they feel that a mask is useful to prevent odors from a patient then this can cause the patient to be offended. Suseno in a general lecture on Ethics philosophy from classical Greek to Javanese people said that Javanese people prioritize "sense" 22 . Some participants said they felt uneasy when doctors used masks.

There was a participant who said that the closeness was reduced because they became less familiar with the doctor, so for the first time the doctor meets the patient, the doctor should open the mask. If the patient already knows the doctor's face then the doctor can wear the mask. The following quotations reflect this dynamic.

"Yes, it affects me. If the doctor uses the mask, we can't see the doctor face. We don't realize one doctor with another. Incidentally, I often meet the doctor when the doctor opens a mask. So, I have been able to distinguish Dr. X with Dr. Y.” (Participant 14)

Patient comfort will be reduced due to the use of masks by health workers, among others because patients cannot see the overall facial expression of the doctor ${ }^{13,14}$. William et al. stated that the length of time a patient knows a doctor will affect the closeness of the patient/ doctor relationship.
Doctors must be able to build a sense of closeness with the patient at the beginning of their meeting so that a sense of connectedness between the patient-doctor is created for the continuation of planned therapy ${ }^{7}$. Some participants said that closeness was not affected by the use of masks because they felt they did not need a long consultation with a doctor. Following the quotation:

"If I come here for KIR, then the doctor's approach is not necessary after that, then it's finished." (Participant 9)

Doctors who can provide lengthy consultation and know how to communicate well with their patients will increase the continuity of the patient's doctor relationship ${ }^{1}$.

Acceptance of a sense of closeness in some participants is not affected by the use of masks because of the patient's concept that masks are worn as required uniforms according to rules that must be adhered to and are a standard of health services, as the following quote states:

"Whatever, maybe the doctors have their own standards." (Participant 5)

Masks are considered as a rule so that their use is proof of obedience to the rules. In a study conducted by Anwar at the Dental Mulut Halimah Hospital, drg. Sikati Makassar stated that of the 45 pediatric patients studied $93.3 \%$ liked doctors who wore white coats and $80 \%$ preferred doctors who wear masks ${ }^{23}$.

There are participants who say communication is not affected by doctor communication because the use of masks is a rule in health services and to maintain the health of doctors. This is consistent with the study of Forgie et al., who found that this was not entirely true. Children and their parents think that masks are natural as well as the white coats worn by doctors. Also, there is the concern that doctors must think about their security too ${ }^{14}$.

Although the concept of masks is not well understood by some patients, the use of masks by doctors is acceptable to these patients and has no effect on closeness. One patient said the mask made him able to communicate comfortably because he did not see the doctor's face, As stated by the following quote:

“We don't know his face. It's covered, isn't it? So, if there are shortcomings, it is not visible."

In Indonesia, Javanese and traditional Sundanese seem to behave similarly to Japanese people, while Batak people are more like Americans. Both Japanese and Javanese consider looking at others as impolite. So, if they bow their heads when speaking, it is intended to respect the other person who is usually a parent or a higher status. Participant 1 has both low education and economic level so they are more likely to feel inferior.

The medical profession is a respected profession. In this study, it was found that doctors are considered someone who can play a more "superior" role than patients because they are considered smarter. According to Hojat in 
several Asian cultures, doctors still hold full authority in therapeutic decisions and override patient input ${ }^{18}$.

Participants with higher education levels such as participants 14 and 15 appreciate the doctor's profession. The oath pledged by a doctor is one guarantee that doctors can be trusted to carry out their duties as well as possible. The public already knows that to become a doctor then someone must recite the doctor's oath first. Not all professions need this, so the doctor's oath is a guarantee that helps the patients believe in the doctor. Sari stated that a noble profession is a medical profession ${ }^{24}$.

Other participants said they trusted their doctor because the doctor explained the details. A detailed description of the doctor's disease, disease process, and treatment plan are needed to increase the patient's trust in the doctor which can eventually support adherence to therapy.

In accordance with what was found in this study the patients' beliefs were not affected by masks as evidenced by the continuity of participants' attendance to the health service on the grounds that they had known the doctor who examined them. For participants who have first time visits, their beliefs are not affected since they come to health services because they already believe in these health services.

\section{Patient Concepts about Masks}

As previously explained, the concept of patients about masks varies according to the background characteristics of patients. Some are conceptualized as rules and are seen as useful in preventing disease transmission so patients can accept doctors who wear masks while serving.

Although understanding the concept of the risk of transmission from the patient to the doctor, some participants suggested wearing a mask for certain times such as when examining patients and not while interviewing/ talking to patients. Long consultations require a feeling of interconnection between patient doctors, as reflected in the following quote:

"Heeh. It is not used. Masks used only when consulting." (Participant 4)

The participants also said that patients who have airborne infectious diseases should wear a mask. However, there is a reluctance to wear masks for reasons that they are unfamiliar, stuffy or uncomfortable with the following quotations:

“... and from patients who are sick too (wearing a mask) ..." (Participant 3)

"Then the patient those who feel that the disease is contagious should use it." (Participant 7)

“... but it feels stifled, so it is removed." (Participant 16)

"If we don't get sick or travel far, we don't get used to wearing masks, we don't get used to it." (Participant 17)

The disadvantages of this study include participants come only from Javanese culture so that we are unable to know the patient acceptance of the use of masks from other regions in Indonesia. Data collection was also only done with in-depth interviews and not followed by observations when patient-doctor communication took place.

\section{CONCLUSIONS}

Based on the results of this study it can be concluded that: (1) Patients who have the concept that masks are a rule for doctors and have benefits in preventing disease; (20 Patients with hearing loss experience poor hearing reception when the doctor speaks using a mask because they cannot see the doctor's lips and face to clarify the conversation; (3) The use of masks affects the patient's closeness but not the patient's beliefs.

\section{Suggestions}

Researchers suggest that doctors who use masks should more often confirm the patient's understanding of ongoing communication between doctors to support the successful management of patients. Similar research is needed for patients from various ethnic groups in Indonesia. In an effort to prevent disease transmission, promotion of the use of appropriate masks should be further improved in addition to the separation of infectious and non-infectious disease services and improvement of hand hygiene.

\section{Acknowledgment}

The authors are grateful to all those who helped complete the research.

\section{Ethical Approval and Informed Consent}

This research has been approved by The Medical Health Research Ethical Committee (MHREC) from Faculty of Medicine Universitas Gadjah Mada, Yogyakarta.

\section{Funding}

Self-funding.

\section{Availability of Data and Material}

Data and material can be accessed via corresponding author.

\section{Conflict of Interest}

None.

\section{REFERENCES}

1. Howie JG, Heaney DJ, Maxwell M, Walker JJ, Freeman GK, Rai H. Quality at general practice consultations: Cross sectional survey. BMJ. 1999 Sep 18;319(7212):738-43

2. Ha JF, Longnecker N. Doctor-patient communication: A review. The Ochsner Journal. 2010 Mar;10(1):38-43.

3. Montague E, Chen PY, Xu J, Chewning B, Barrett B. Nonverbal interpersonal interactions in clinical encounters and patient perceptions of empathy. J Participat Med. 2013 Aug 14;5:e33.

4. Kusnanto H. Patient-Centered Care. Review of Primary Care Practice and Education (Kajian Praktik dan Pendidikan Layanan Primer). 2018;1(2):51-2.

5. Khan TM, Hassali MA, Al-Haddad MS. Patient-physician communication barrier: A pilot study evaluating patient experiences. Journal of Young Pharmacists. 2011 Jul 1;3(3):250-5.

6. Stewart M, Brown JB, Boon H, Galajda J, Meredith L, Sangster M. Evidence on patient-doctor communication. Cancer. 1999;25(1999):30.

7. Williams S, Weinman J, Dale J. Doctor-patient communication and patient satisfaction. Fam Pract. 1998;15(5):480-92. 
8. Rezaei F, Askari HA. Checking the relationship between physicians' communication skills and outpatients' satisfaction in the clinics of Isfahan Al-Zahra (S) Hospital in 2011. Journal of Education and Health Promotion. 2014;3.

9. Suchman AL, Markakis K, Beckman HB, Frankel R. A model of empathic communication in the medical interview. Jama. $1997 \mathrm{Feb}$ 26;277(8):678-82.

10. Wong KMC, Yip KHB, Mercer S, Griffiths S, Kung K, Wong CSM, et al. Effect of facemasks on empathy and relational continuity: A randomised controlled trial in primary care. BMC Family Practice. 2013;14:200 (http://www.biomedcentral. com/1471-2296/14/200)

11. Centers for Disease Control and Prevention (CDC). Guide to infection prevention for outpatient settings: Minimum expectations for safe care. 2014

12. Harwanti N. Personal protective equipment usage in providing protection for workers in instalasi inpatient I RSUP dr. Sardjito Yogyakarta [Doctoral dissertation]. Surakarta: Universitas Sebelas Maret. 2009.

13. Mufarida A. The effect of nurse therapeutic communication implementation on patient satisfaction levels (case study in inpatient class 3 patients of dr. Soebandi Jember Hospital). 2011.

14. Forgie SE, Reitsma J, Spady D, Wright B, Stobart K. The "fear factor" for surgical masks and face shields, as perceived by children and their parents. Pediatrics. 2009 Oct 1;124(4):e777-81.

15. Naomi P. Measurement of customer satisfaction level on health care services (using performance importance analysis method in inpatient of Sumedang Hospital). Abmas. 2005;77.

16. Potter P, Perry A. Fundamental textbooks for nursing concepts, processes, and practices. 4th ed. Jakarta: EGC. 2005

17. Kurniawan AP, Hasanat NU. Differences in emotional expression at several generations of Javanese in Yogyakarta. Journal of Psychology. 2007;34(1):1-7.

18. Hojat M. Empathy in patient care: antecedents, development, measurement, and outcomes. Springer Science \& Business Media. 2007 Nov 12.

19. Ong LM, De Haes JC, Hoos AM, Lammes FB. Doctor-patient communication: A review of the literature. Social Science \& Medicine. 1995 Apr 1;40(7):903-18

20. Basuki E. Communication among health workers. Indonesian Medical Magazine. 2008;58(9):340-5.

21. Manoppo HB. Study of interpersonal communication at the Bethesda Tomohon Hospital outpatient / obstetric clinic. JURNAL ACTA DIURNA. 2014 Aug 8;3(2)

22. Suseno, FM. The fourth paper is a general lecture on ethical philosophy from classical Greece to Java at Teater Salihara. Jakarta. February 232013.

23. Anwar AI. Children's perception of their dentist at Halimah Dg. Sikati dental hospital in Makassar. Journal of Dentomaxillofacial Science. 2014 Jan 1;13(2):91-4.

24. Sari DP. What is the meaning of "profit" for the doctor's profession? Multiparadigm Accounting Journal. 2014;5(1):130-8. 